\title{
On cycle inequalities for convex-star bodies
}

\author{
Chang-Jian Zhao ${ }^{\mathrm{a}, *}$, Wing-Sum Cheung ${ }^{\mathrm{b}}$ \\ ${ }^{a}$ Department of Mathematics, China Jiliang University, Hangzhou 310018, P. R. China. \\ ${ }^{b}$ Department of Mathematics, The University of Hong Kong, Pokfulam Road, Hong Kong. \\ Communicated by $R$. Saadati
}

\begin{abstract}
In this paper, we establish new cycle inequalities for convex-star bodies, which are joint improvements of the cycle inequality for convex bodies and the dual cycle inequality for star bodies. (c)2017 All rights reserved.

Keywords: Convex body, star body, mixed volume, dual mixed volume, the cycle inequality, the dual cycle inequality. 2010 MSC: 52A40, 26D15.
\end{abstract}

\section{Introduction}

It is well-known that the cycle inequality for convex bodies can be stated below (see [6, p.401]). If $K$ is convex body and $0 \leqslant i<j<k \leqslant n$, then

$$
W_{j}(K)^{k-i} \geqslant W_{i}(K)^{k-j} W_{k}(K)^{j-i},
$$

with equality if and only if $\mathrm{K}$ is an $\mathrm{n}$-ball centered at the origin.

Here, $W_{i}(K)$ denotes the quermassintegral of convex body $K$, i.e., $W_{i}(K)=V(\underbrace{K, \ldots, K}_{n-i}, \underbrace{B, \ldots, B}_{i})$, the letter B stands for the unit ball centered at the origin, and $V\left(K_{1}, \ldots, K_{n}\right)$ is the mixed volume of convex bodies $\mathrm{K}_{1}, \ldots, \mathrm{K}_{\mathrm{n}}$, defined by (see e.g. [1])

$$
V\left(K_{1}, \ldots, K_{n-1}, K\right)=\frac{1}{n} \int_{S^{n-1}} h(K, u) d S\left(K_{1}, \ldots, K_{n-1} ; u\right) .
$$

Here $S^{n-1}$ denotes the unit sphere in $\mathbb{R}^{n}$, and

$$
h(K, u)=\max \left\{u \cdot x: x \in K, u \in S^{n-1}\right\}
$$

denotes the support function of $K$, and $u \cdot x$ denotes the usual inner product $u$ and $x$ in $\mathbb{R}^{n}$, and where $\mathrm{S}\left(\mathrm{K}_{1}, \ldots, \mathrm{K}_{\mathrm{n}-1} ; \cdot\right)$ is a Borel measure, called the mixed surface area measure of $\mathrm{K}_{1}, \ldots, \mathrm{K}_{\mathrm{n}-1}$ (see [4]). In fact, the measure $S\left(K_{1}, \ldots, K_{n-1} ; \cdot\right)$ can be defined by (1.2) for all convex bodies $K$.

\footnotetext{
*Corresponding author

Email addresses: chjzhao@163.com, chjzhao@aliyun.com (Chang-Jian Zhao), wscheung@hku.hk (Wing-Sum Cheung)
} doi:10.22436/jnsa.010.09.30 
The classical dual cycle inequality for star bodies can be stated below (see [3] or [2]). If $K$ is star body and $i<j<k$, then

$$
\widetilde{W}_{j}(K)^{k-i} \leqslant \widetilde{W}_{i}(K)^{k-j} \widetilde{W}_{k}(K)^{j-i}
$$

with equality if and only if $K$ is an $n$-ball centered at the origin.

Here, $\widetilde{W}_{i}(K)$ denotes the dual quermassintegral of star body $K$, i.e., $\widetilde{W}_{i}(K)=\widetilde{V}(\underbrace{K}_{n-i}, \ldots, K, \underbrace{B, \ldots, B}_{i})$, and $\widetilde{V}\left(K_{1}, \ldots, K_{n}\right)$ is the dual mixed volume of star bodies $K_{1}, \ldots, K_{n}$, defined by (see e.g., [3])

$$
\widetilde{V}\left(K_{1}, \ldots, K_{n}\right)=\frac{1}{n} \int_{S^{n-1}} \rho\left(K_{1}, u\right) \cdots \rho\left(K_{n}, u\right) d S(u),
$$

where

$$
\rho(K, u)=\max \left\{\lambda \geqslant 0: \lambda u \in K, u \in S^{n-1}\right\}
$$

is the radial function of star body $\mathrm{K}$.

The aim of this paper is to establish mixed cycle inequalities for convex and star bodies, which are joint improvements of the cycle inequality and the dual cycle inequality.

Theorem 1.1. Let $\mathrm{K}$ be convex body, and $\mathrm{D}$ star body in $\mathbb{R}^{\mathrm{n}}$, and $\mathrm{D} \subseteq \mathrm{K}$. If $0 \leqslant \mathrm{i}<\mathrm{j}<\mathrm{k} \leqslant \mathrm{n}$, then

$$
\left(W_{j}(K)-\widetilde{W}_{j}(D)\right)^{k-i} \geqslant\left(W_{i}(K)-\widetilde{W}_{i}(D)\right)^{k-j}\left(W_{k}(K)-\widetilde{W}_{k}\left(D^{\prime}\right)\right)^{j-i},
$$

with equality if and only if $\mathrm{K}$ and $\mathrm{D}$ are $\mathrm{n}$-balls centered at the origin.

Remark 1.2. Let D be single point in (1.4), then (1.4) becomes the cycle inequality (1.1) for convex bodies.

Theorem 1.1 is just a special case of Theorem 3.6 established in Section 3.

Theorem 1.3. Let $K$ be star body, and $D$ convex body in $\mathbb{R}^{n}$ such that $\widetilde{W}_{m}(K) \geqslant W_{m}(D), m=i, j, k$. If $0 \leqslant i<j<k \leqslant n$, then

$$
\left(\widetilde{W}_{j}(K)-W_{j}(D)\right)^{k-i} \leqslant\left(\widetilde{W}_{i}(K)-W_{i}(D)\right)^{k-j}\left(\widetilde{W}_{k}(K)-W_{k}\left(D^{\prime}\right)\right)^{j-i},
$$

with equality if and only if $\mathrm{K}$ and $\mathrm{D}$ are $\mathrm{n}$-balls centered at the origin.

Remark 1.4. Let D be single point in (1.5), then (1.5) becomes the dual cycle inequality (1.3) for star bodies.

Theorem 1.3 just is a special case of Theorem 3.9 established in Section 3.

In the following, we also establish cycle inequality for sum of the dual quermassintegrals of star bodies.

Theorem 1.5. Let $\mathrm{K}$ and $\mathrm{D}$ be star bodies in $\mathbb{R}^{\mathrm{n}}$. If $\mathrm{i}<\mathrm{j}<\mathrm{k}$, then

$$
\left(\widetilde{W}_{j}(K)+\widetilde{W}_{j}(D)\right)^{k-i} \leqslant\left(\widetilde{W}_{i}(K)+\widetilde{W}_{i}(D)\right)^{k-j}\left(\widetilde{W}_{k}(K)+\widetilde{W}_{k}\left(D^{\prime}\right)\right)^{j-i},
$$

with equality if and only if $\mathrm{K}$ and $\mathrm{D}$ are $\mathrm{n}$-balls centered at the origin.

Remark 1.6. Let $\mathrm{K}$ or $\mathrm{D}$ be single point in (1.6), then (1.6) becomes the dual cycle inequality (1.3) for star bodies.

Theorem 1.5 just is a special case of Theorem 3.10 established in Section 3.

\section{Notations and definitions}

The setting for this paper is $n$-dimensional Euclidean space $\mathbb{R}^{n}$. Let $\mathcal{K}^{n}$ denote the set of convex bodies (compact, convex subsets with non-empty interiors) in $\mathbb{R}^{\mathfrak{n}}$. We reserve the letter $u$ for unit vectors. The surface of $B$ is $S^{n-1}$. We use $V(K)$ for the $n$-dimensional volume of convex body $K$. 
Let $\delta$ denote the Hausdorff metric on $\mathcal{K}^{n}$, i.e., for $K, L \in \mathcal{K}^{n}$,

$$
\delta(K, L)=|h(K, u)-h(L, u)|_{\infty},
$$

where $|\cdot|_{\infty}$ denotes the sup-norm on the space of continuous functions $C\left(S^{n-1}\right)$ (see, e.g., [6]).

Associated with a compact subset $K$ of $\mathbb{R}^{n}$, which is star-shaped with respect to the origin. If $\rho(K, \cdot)$ is positive and continuous, $\mathrm{K}$ will be called a star body. Let $\mathcal{S}^{n}$ denote the set of star bodies in $\mathbb{R}^{n}$. Let $\tilde{\delta}$ denote the radial Hausdorff metric as follows, if $K, L \in \mathcal{S}^{n}$, then (see, e.g., [2])

$$
\tilde{\delta}(K, L)=|\rho(K, u)-\rho(L, u)|_{\infty} .
$$

\section{Mixed volumes}

If $K_{i} \in \mathcal{K}^{n}(i=1,2, \ldots, r)$ and $\lambda_{i}(i=1,2, \ldots, r)$ are nonnegative real numbers, then of fundamental importance is the fact that the volume of $\sum_{i=1}^{r} \lambda_{i} K_{i}$ is a homogeneous polynomial in $\lambda_{i}$ given by (see e.g., [1])

$$
V\left(\sum_{i=1}^{r} \lambda_{i} K_{i}\right)=\sum_{i_{1}, \ldots, i_{n}} \lambda_{i_{1}} \ldots \lambda_{i_{n}} V_{i_{1} \ldots i_{n}}
$$

where the sum is taken over all $n$-tuples $\left(i_{1}, \ldots, i_{n}\right)$ of positive integers not exceeding $r$. The coefficient $V_{i_{1}, \ldots, i_{n}}$ depends only on the bodies $K_{i_{1}}, \ldots, K_{i_{n}}$, and is uniquely determined by (2.1), it is called the mixed volume of $K_{i_{1}}, \ldots, K_{i_{n}}$, and is written as $V\left(K_{i_{1}}, \ldots, K_{i_{n}}\right)$. Let $K_{1}=\cdots=K_{n-i}=K$ and $K_{n-i+1}=\cdots=$ $K_{n}=L$, then the mixed volume $V\left(K_{1}, \ldots, K_{n}\right)$ is usually written $V_{i}(K, L)$. If $L=B$, then $V_{i}(K, B)$ is the ith projection measure (quermassintegral) of $K$ and is written as $W_{i}(K)$.

\subsection{Dual mixed volumes}

If $x_{1}, \ldots, x_{r} \in \mathbb{R}^{n}$, then $x_{1} \tilde{+} \ldots \tilde{+} x_{r}$ is defined to be the usual vector sum of $x_{1}, \ldots, x_{r}$, provided $x_{1}, \ldots, x_{r}$ all lie in a 1-dimensional subspace of $\mathbb{R}^{n}$, and as the zero vector otherwise.

If $\mathrm{K}_{1}, \ldots, \mathrm{K}_{\mathrm{r}} \in \mathcal{S}^{n}$ and $\lambda_{1}, \ldots, \lambda_{\mathrm{r}} \in \mathbb{R}$, then their radial Minkowski linear combination, is defined by $\lambda_{1} K_{1} \tilde{+} \cdots \tilde{+} \lambda_{r} K_{r}=\left\{\lambda_{1} x_{1} \tilde{+} \cdots \tilde{+} \lambda_{r} x_{r}: x_{i} \in K_{i}\right\}$. For $K_{1}, \ldots, K_{r} \in \mathcal{S}^{n}$ and $\lambda_{1}, \ldots, \lambda_{r} \geqslant 0$, the volume of the radial Minkowski linear combination $\lambda_{1} K_{1} \tilde{+} \ldots \tilde{+} \lambda_{r} K_{r}$ is a homogeneous $n$ th-degree polynomial in the $\lambda_{i}$,

$$
\mathrm{V}\left(\lambda_{1} \mathrm{~K}_{1} \tilde{+} \ldots \tilde{+} \lambda_{\mathrm{r}} \mathrm{K}_{\mathrm{r}}\right)=\sum \widetilde{\mathrm{V}}_{i_{1}, \ldots, i_{n}} \lambda_{i_{1}} \cdots \lambda_{i_{n}}
$$

where the sum is taken over all $n$-tuples $\left(i_{1}, \ldots, i_{n}\right)$ whose entries are positive integers not exceeding $r$. If we require the coefficients of the polynomial in the above identity to be symmetric in their arguments, then they are uniquely determined by (2.2). The coefficient $\widetilde{\mathrm{V}}_{i_{1}, \ldots, i_{n}}$ is nonnegative and depends only on the bodies $K_{i_{1}}, \ldots, K_{i_{n}}$. It is written as $\widetilde{V}\left(K_{i_{1}}, \ldots, K_{i_{n}}\right)$ and is called the dual mixed volume of $K_{i_{1}}, \ldots, K_{i_{n}}$. If $K_{1}=\cdots=K_{n-i}=K, K_{n-i+1}=\cdots=K_{n}=L$, the dual mixed volume is written as $\widetilde{V}_{i}(K, L)$. The dual mixed volume $\widetilde{V}_{i}(K, B)$ is written as $\widetilde{W}_{i}(K)$ and is called dual quermassintegral of star body $K$ (see e.g., [2]).

\section{The cycle inequalities for convex-star bodies}

Lemma 3.1 ([6, p.401]). If $K, L \in \mathcal{K}^{n}$, and $0 \leqslant i<j<k \leqslant n$, then

$$
V_{j}(K, L)^{k-i} \geqslant V_{k}(K, L)^{j-i} V_{i}(K, L)^{k-j},
$$

with equality if and only if $\mathrm{K}$ and $\mathrm{L}$ are homothetic. 
Lemma 3.2 ([3]). If $\mathrm{K}, \mathrm{L} \in \mathcal{S}^{n}$ and $i<j<k$, then

$$
\widetilde{V}_{j}(K, L)^{k-i} \leqslant \widetilde{V}_{k}(K, L)^{j-i} \widetilde{V}_{i}(K, L)^{k-j},
$$

with equality if and only if $\mathrm{K}$ and $\mathrm{L}$ are dilates.

Lemma 3.3 ([3]). If $K, L \in \mathcal{K}^{n}$, then for $0<i<n$,

$$
\widetilde{V}_{i}(K, L) \leqslant V_{i}(K, L),
$$

with equality if and only if $\mathrm{K}$ and $\mathrm{L}$ are dilates.

Lemma 3.4 ([5, p.58], Popoviciu's inequality). Let $p>0, q>0,1 / p+1 / q=1$, and $a=\left\{a_{1}, \ldots, a_{n}\right\}$ and $\mathrm{b}=\left\{\mathrm{b}_{1}, \ldots, \mathrm{b}_{\mathrm{n}}\right\}$ be two series of positive real numbers such that $\mathrm{a}_{1}^{\mathrm{p}}-\sum_{i=2}^{n} a_{i}^{p}>0$ and $b_{1}^{q}-\sum_{i=2}^{n} b_{i}^{q}>0$. Then

$$
\left(a_{1}^{p}-\sum_{i=2}^{n} a_{i}^{p}\right)^{1 / p}\left(b_{1}^{q}-\sum_{i=2}^{n} b_{i}^{q}\right)^{1 / q} \leqslant a_{1} b_{1}-\sum_{i=2}^{n} a_{i} b_{i},
$$

with equality if and only if $\mathrm{a}=\mu \mathrm{b}$, where $\mu$ is a constant.

Lemma 3.5 ([9]). Let $\mathrm{a}, \mathrm{b} \geqslant 0, \mathrm{c}, \mathrm{d}>0,0<\alpha<1,0<\beta<1$ and $\alpha+\beta=1$, then

$$
a^{\alpha} c^{\beta}+b^{\alpha} d^{\beta} \leqslant(a+b)^{\alpha}(c+d)^{\alpha},
$$

with equality if and only if $\mathrm{a} / \mathrm{b}=\mathrm{c} / \mathrm{d}$.

Theorem 3.6. Let $\mathrm{K}, \mathrm{L} \in \mathcal{K}^{n}, \mathrm{D}, \mathrm{D}^{\prime} \in \mathcal{S}^{n}, \mathrm{D} \subseteq \mathrm{K}$, and $\mathrm{D}^{\prime} \subseteq \mathrm{L}$. If $0 \leqslant i<j<\mathrm{k} \leqslant \mathrm{n}$, then

$$
\left(V_{j}(K, L)-\widetilde{V}_{j}\left(D, D^{\prime}\right)\right)^{k-i} \geqslant\left(V_{k}(K, L)-\widetilde{V}_{k}\left(D, D^{\prime}\right)\right)^{j-i}\left(V_{i}(K, L)-\widetilde{V}_{i}\left(D, D^{\prime}\right)\right)^{k-j}
$$

with equality if and only if $\mathrm{K}$ and $\mathrm{L}$ are homothetic, $\mathrm{D}$ and $\mathrm{D}^{\prime}$ are dilates, and $\left(\mathrm{V}_{\mathrm{k}}(\mathrm{K}, \mathrm{L}), \widetilde{\mathrm{V}}_{\mathrm{k}}\left(\mathrm{D}, \mathrm{D}^{\prime}\right)\right)=\mu\left(\mathrm{V}_{\mathrm{i}}(\mathrm{K}, \mathrm{L})\right.$, $\left.\widetilde{V}_{i}\left(D, D^{\prime}\right)\right)$, where $\mu$ is a constant.

Proof. From Lemmas 3.1 and 3.2, for $K, L \in \mathcal{K}^{n}$ and $0 \leqslant i<j<k \leqslant n$

$$
V_{j}(K, L)^{k-i} \geqslant V_{k}(K, L)^{j-i} V_{i}(K, L)^{k-j},
$$

with equality if and only if $K$ and $L$ are homothetic, and for $D, D^{\prime} \in \mathcal{S}^{n}$ and $i<j<k$

$$
\widetilde{V}_{j}\left(D, D^{\prime}\right)^{k-i} \leqslant \widetilde{V}_{k}\left(D, D^{\prime}\right)^{j-i} \widetilde{V}_{i}\left(D, D^{\prime}\right)^{k-j},
$$

with equality if and only if $D$ and $D^{\prime}$ are dilates. Hence for $0 \leqslant i<j<k \leqslant n$,

$$
\begin{aligned}
& V_{j}(K, L)-\widetilde{V}_{j}\left(D, D^{\prime}\right) \\
& \geqslant V_{k}(K, L)^{(j-i) /(k-i)} V_{i}(K, L)^{(k-j) /(k-i)}-\widetilde{V}_{k}\left(D, D^{\prime}\right)^{(j-i) /(k-i)} \widetilde{V}_{i}\left(D, D^{\prime}\right)^{(k-j) /(k-i)},
\end{aligned}
$$

with equality if and only if $\mathrm{K}$ and $\mathrm{L}$ are homothetic, and $\mathrm{D}$ and $\mathrm{D}^{\prime}$ are dilates.

Noticing that $\mathrm{D} \subseteq \mathrm{K}$ and $\mathrm{D}^{\prime} \subseteq \mathrm{L}$, and from (3.1), we have

$$
V_{k}(K, L) \geqslant \widetilde{V}_{k}(K, L) \geqslant \widetilde{V}_{k}\left(D, D^{\prime}\right),
$$

and

$$
V_{i}(K, L) \geqslant \widetilde{V}_{i}(K, L) \geqslant \widetilde{V}_{i}\left(D, D^{\prime}\right)
$$


Noting that (3.5), (3.6), and (3.7), by using (3.2) in Lemma 3.4, we obtain

$$
\left(V_{j}(K, L)-\widetilde{V}_{j}\left(D, D^{\prime}\right)\right)^{k-i} \geqslant\left(V_{k}(K, L)-\widetilde{V}_{k}\left(D, D^{\prime}\right)\right)^{j-i}\left(V_{i}(K, L)-\widetilde{V}_{i}\left(D, D^{\prime}\right)\right)^{k-j}
$$

with equality if and only if $K$ and $L$ are homothetic, $D$ and $D^{\prime}$ are dilates, and $\left(V_{k}(K, L), \widetilde{V}_{k}\left(D, D^{\prime}\right)\right)=$ $\mu\left(V_{i}(K, L), \widetilde{V}_{i}\left(D, D^{\prime}\right)\right)$, where $\mu$ is a constant.

Remark 3.7. Putting $L=D^{\prime}=B$ in (3.4), then (3.4) becomes the cycle inequality (1.4) for the mixed volumes difference stated in the introduction.

Putting $i=0, j=n-i$ and $k=n$ in (3.4), we have the following.

Corollary 3.8. Let $\mathrm{K}, \mathrm{L} \in \mathcal{K}^{\mathrm{n}}, \mathrm{D}, \mathrm{D}^{\prime} \in \mathcal{S}^{\mathrm{n}}$, and $\mathrm{D} \subseteq \mathrm{K}$ and $\mathrm{D}^{\prime} \subseteq \mathrm{L}$. If $0<\mathrm{i}<\mathrm{n}$, then

$$
\left(V_{i}(L, K)-\widetilde{V}_{i}\left(D^{\prime}, D\right)\right)^{n} \geqslant\left(V(L)-V\left(D^{\prime}\right)\right)^{n-i}(V(K)-V(D))^{i}
$$

with equality if and only if $\mathrm{K}$ and $\mathrm{L}$ are homothetic, $\mathrm{D}$ and $\mathrm{D}^{\prime}$ are dilates, and $\left(\mathrm{V}(\mathrm{L}), \mathrm{V}\left(\mathrm{D}^{\prime}\right)\right)=\mu(\mathrm{V}(\mathrm{K}), \mathrm{V}(\mathrm{D}))$, where $\mu$ is a constant.

Putting $i=1$ in (3.8), (3.8) becomes

$$
\left(\mathrm{V}_{1}(\mathrm{~L}, \mathrm{~K})-\widetilde{\mathrm{V}}_{1}\left(\mathrm{D}^{\prime}, \mathrm{D}\right)\right)^{\mathrm{n}} \geqslant\left(\mathrm{V}(\mathrm{L})-\mathrm{V}\left(\mathrm{D}^{\prime}\right)\right)^{\mathrm{n}-1}(\mathrm{~V}(\mathrm{~K})-\mathrm{V}(\mathrm{D}))
$$

with equality if and only if $K$ and $L$ are homothetic, $D$ and $D^{\prime}$ are dilates, and $\left(V_{k}(K, L), \widetilde{V}_{k}\left(D, D^{\prime}\right)\right)=$ $\mu\left(V_{i}(K, L), \widetilde{V}_{i}\left(D, D^{\prime}\right)\right)$, where $\mu$ is a constant.

Let $\mathrm{D}$ and $\mathrm{D}^{\prime}$ be single point in (3.9), then (3.9) becomes the classical Minkowski inequality for convex bodies: if $K, L \in \mathcal{K}^{n}$, then

$$
V_{1}(L, K)^{n} \geqslant V(L)^{n-1} V(K),
$$

with equality if and only if $K$ and $L$ are homothetic.

Theorem 3.9. Let $\mathrm{K}, \mathrm{L} \in \mathcal{S}^{n}, \mathrm{D}, \mathrm{D}^{\prime} \in \mathcal{K}^{\mathrm{n}}$ such that $\widetilde{\mathrm{V}}_{\mathrm{m}}(\mathrm{K}, \mathrm{L}) \geqslant \mathrm{V}_{\mathrm{m}}\left(\mathrm{D}, \mathrm{D}^{\prime}\right)$ and $m=i, j, k$. If $0 \leqslant i<j<k \leqslant$ $n$, then

$$
\left(\widetilde{V}_{j}(K, L)-V_{j}\left(D, D^{\prime}\right)\right)^{k-i} \leqslant\left(\widetilde{V}_{k}(K, L)-V_{k}\left(D, D^{\prime}\right)\right)^{j-i}\left(\widetilde{V}_{i}(K, L)-V_{i}\left(D, D^{\prime}\right)\right)^{k-j}
$$

with equality if and only if $\mathrm{K}$ and $\mathrm{L}$ are dilates, $\mathrm{D}$ and $\mathrm{D}^{\prime}$ are homothetic, and $\left(\widetilde{\mathrm{V}}_{\mathrm{k}}(\mathrm{K}, \mathrm{L}), \mathrm{V}_{\mathrm{k}}\left(\mathrm{D}, \mathrm{D}^{\prime}\right)\right)=\mu\left(\widetilde{\mathrm{V}}_{\mathrm{i}}(\mathrm{K}, \mathrm{L})\right.$, $\left.\mathrm{V}_{i}\left(\mathrm{D}, \mathrm{D}^{\prime}\right)\right)$, where $\mu$ is a constant.

The proof of Theorem 3.9 can be completed by following the same steps as in the proof of Theorem 3.6 with suitable changes and note the use of reverse Popoviciu's inequality instead of using Popoviciu's inequality. Here, we omit the details.

Putting $L=D^{\prime}=B$ in (3.10), (3.10) becomes the cycle inequality (1.5) for the dual quermassintegrals difference stated in the introduction.

Theorem 3.10. If $\mathrm{K}, \mathrm{L}, \mathrm{D}, \mathrm{D}^{\prime} \in \mathcal{S}^{n}$ and $i<j<k$, then

$$
\left(\widetilde{V}_{j}(K, L)+\widetilde{V}_{j}\left(D, D^{\prime}\right)\right)^{k-i} \leqslant\left(\widetilde{V}_{k}(K, L)+\widetilde{V}_{k}\left(D, D^{\prime}\right)\right)^{j-i}\left(\widetilde{V}_{i}(K, L)+\widetilde{V}_{i}\left(D, D^{\prime}\right)\right)^{k-j}
$$

with equality if and only if $\mathrm{K}$ and $\mathrm{L}$ are dilates, $\mathrm{D}$ and $\mathrm{D}^{\prime}$ are dilates, and

$$
\left(\widetilde{V}_{k}(K, L), \widetilde{V}_{k}\left(D, D^{\prime}\right)\right)=\mu\left(\widetilde{V}_{i}(K, L), \widetilde{V}_{i}\left(D, D^{\prime}\right)\right),
$$

where $\mu$ is a constant. 
Proof. From Lemma 3.2, for K, L, D, $\mathrm{D}^{\prime} \in \mathcal{S}^{n}$ and $i<j<k$, we have

$$
\widetilde{V}_{j}(K, L)^{k-i} \leqslant \widetilde{V}_{k}(K, L)^{j-i} \widetilde{V}_{i}(K, L)^{k-j},
$$

with equality if and only if $K$ and $L$ are dilates, and

$$
\widetilde{V}_{j}\left(D, D^{\prime}\right)^{k-i} \leqslant \widetilde{V}_{k}\left(D, D^{\prime}\right)^{j-i} \widetilde{V}_{i}\left(D, D^{\prime}\right)^{k-j},
$$

with equality if and only if $D$ and $D^{\prime}$ are dilates. Hence for $i<j<k$,

$$
\begin{aligned}
& \widetilde{V}_{j}(K, L)+\widetilde{V}_{j}\left(D, D^{\prime}\right) \\
& \quad \leqslant \widetilde{V}_{k}(K, L)^{(j-i) /(k-i)} \widetilde{V}_{i}(K, L)^{(k-j) /(k-i)}+\widetilde{V}_{k}\left(D, D^{\prime}\right)^{(j-i) /(k-i)} \widetilde{V}_{i}\left(D, D^{\prime}\right)^{(k-j) /(k-i),}
\end{aligned}
$$

with equality if and only if $\mathrm{K}$ and $\mathrm{L}$ are dilates, and $\mathrm{D}$ and $\mathrm{D}^{\prime}$ are dilates.

Noting that (3.12), by using (3.3), we obtain

$$
\left(\widetilde{V}_{j}(K, L)+\widetilde{V}_{j}\left(D, D^{\prime}\right)\right)^{k-i} \leqslant\left(\widetilde{V}_{k}(K, L)+\widetilde{V}_{k}\left(D, D^{\prime}\right)\right)^{j-i}\left(\widetilde{V}_{i}(K, L)+\widetilde{V}_{i}\left(D, D^{\prime}\right)\right)^{k-j}
$$

with equality if and only if $K$ and $L$ are dilates, $D$ and $D^{\prime}$ are dilates, and $\left(\widetilde{V}_{k}(K, L), \widetilde{V}_{k}\left(D, D^{\prime}\right)\right)=$ $\mu\left(\widetilde{V}_{i}(K, L), \widetilde{V}_{i}\left(D, D^{\prime}\right)\right)$, where $\mu$ is a constant.

Remark 3.11. Putting $L=D^{\prime}=B$ in (3.11), then (3.11) becomes the cycle inequality (1.6) for dual mixed volumes sum stated in the introduction.

Putting $i=0, j=n-i$, and $k=n$ in (3.11), we have the following.

Corollary 3.12. Let $\mathrm{K}, \mathrm{L}, \mathrm{D}, \mathrm{D}^{\prime} \in \mathcal{S}^{\mathrm{n}}$. If $\mathrm{i}<\mathrm{n}-1$, then

$$
\left(\widetilde{V}_{i}(L, K)+\widetilde{V}_{i}\left(D^{\prime}, D\right)\right)^{n} \leqslant\left(V(L)+V\left(D^{\prime}\right)\right)^{n-i}(V(K)+V(D))^{i}
$$

with equality if and only if $\mathrm{K}$ and $\mathrm{L}$ are dilates, $\mathrm{D}$ and $\mathrm{D}^{\prime}$ are dilates, and $\left(\mathrm{V}(\mathrm{L}), \mathrm{V}\left(\mathrm{D}^{\prime}\right)\right)=\mu(\mathrm{V}(\mathrm{K}), \mathrm{V}(\mathrm{D}))$, where $\mu$ is a constant.

Putting $i=1$ in (3.13), (3.13) becomes

$$
\left(\widetilde{\mathrm{V}}_{1}(\mathrm{~L}, \mathrm{~K})+\widetilde{\mathrm{V}}_{1}\left(\mathrm{D}^{\prime}, \mathrm{D}\right)\right)^{\mathrm{n}} \leqslant\left(\mathrm{V}(\mathrm{L})+\mathrm{V}\left(\mathrm{D}^{\prime}\right)\right)^{\mathrm{n}-1}(\mathrm{~V}(\mathrm{~K})+\mathrm{V}(\mathrm{D}))
$$

with equality if and only if $K$ and $L$ are dilates, $D$ and $D^{\prime}$ are dilates, and $\left(V(L), V\left(D^{\prime}\right)\right)=\mu(V(K), V(D))$, where $\mu$ is a constant.

Let $\mathrm{D}$ and $\mathrm{D}^{\prime}$ be single point in (3.14), (3.14) becomes the classical dual Minkowski inequality for convex bodies: if $\mathrm{K}, \mathrm{L} \in \mathcal{S}^{n}$, then

$$
\widetilde{V}_{1}(L, K)^{n} \leqslant V(L)^{n-1} V(K),
$$

with equality if and only if $K$ and $L$ are dilates.

What is worth mentioning here is that in recent years, the study of new type of cyclic inequalities has become a hot issue. For references to the study of the cyclic Brunn-Minkowski inequalities, please see [8] and [7].

\section{Acknowledgment}

The research of first author is supported by the Natural Science Foundation of China (11371334). Research of the second author is supported by a HKU Seed Grant for Basic Research. 


\section{References}

[1] Y. D. Burago, V. A. Zalgaller, Geometric inequalities, Translated from the Russian by A. B. Sosinskiǔ, Grundlehren der Mathematischen Wissenschaften [Fundamental Principles of Mathematical Sciences], Springer Series in Soviet Mathematics, Springer-Verlag, Berlin, (1988). 1, 2

[2] R. J. Gardner, Geometric tomography, Encyclopedia of Mathematics and its Applications, Cambridge University Press, Cambridge, (1996). 1, 1, 2.1

[3] E. Lutwak, Dual mixed volumes, Pacific J. Math., 58 (1975), 531-538. 1, 1, 3.2, 3.3

[4] E. Lutwak, The Brunn-Minkowski-Firey theory, I, Mixed volumes and the Minkowski problem, J. Differential Geom., 38 (1993), 131-150. 1

[5] D. S. Mitrinović, Analytic inequalities, In cooperation with P. M. Vasi. Die Grundlehren der mathematischen Wissenschaften, Band 165 Springer-Verlag, New York-Berlin, (1970). 3.4

[6] R. Schneider, Convex bodies: the Brunn-Minkowski theory, Second expanded edition, Encyclopedia of Mathematics and its Applications, Cambridge University Press, Cambridge, (2014). 1, 1, 3.1

[7] C.-J. Zhao, Dual cyclic Brunn-Minkowski inequalities, Bull. Belg. Math. Soc. Simon Stevin, 22 (2015), 391-401. 3

[8] C.-J. Zhao, Cyclic Brunn-Minkowski inequalities for p-affine surface area, Quaest. Math., 40 (2017), 467-476. 3

[9] C.-J. Zhao, G.-S. Leng, Inequalities for dual quermassintegrals of mixed intersection bodies, Proc. Indian Acad. Sci. Math. Sci., 115 (2005), 79-91. 3.5 